\title{
PERSPECTIVAS EN LA FORMACIÓN DE EDUCADORES AMBIENTALES Y EL CONOCIMIENTO DIDÁCTICO
}

\author{
PERSPECTIVAS NA FORMAÇÃO DE \\ EDUCADORES AMBIENTAIS E O \\ CONHECIMENTO DIDÁTICO
}

\author{
Leidy Gabriela Ariza Ariza ${ }^{1}$ \\ José Vicente de Freitas²
}

Resumen: El texto presenta la relación entre las publicaciones respecto a la formación de educadores ambientales y el Conocimiento Didáctico del Contenido (CDC) en educación ambiental. En la formación de educadores ambientales y las relaciones establecidas para la generación de propuestas educativas alrededor del conocimiento específico. Como resultado se identificaron categorías socioculturales, políticas, éticas, contexto, epistemológicas, curriculares, entre otras, alrededor del educador ambiental, e intereses de investigación. Se destaca la necesidad de profundizar en el CDC para identificar los indicios relacionados con la formación de educadores de acuerdo a las necesidades del conocimiento y a las preocupaciones de la comunidad que hace parte y su campo de acción.

Palabras Clave: Formación; Educador Ambiental; Conocimiento Didáctico del Contenido; Educación ambiental. 
Resumo: O texto apresenta a relação entre as publicações sobre a formação de educadores ambientais e o Conhecimento Didático do Conteúdo (CDC) na educação ambiental. Na formação de educadores ambientais e as relações por pesquisadores para gerar propostas educativas mediante o conhecimento específico. Como resultado, identifica categorias socioculturais, políticas, éticas, contextuais, epistemológicas, curriculares, entre outras, em torno do educador ambiental e interesses de investigação. Destaca a necessidade de se aprofundar no CDC para identificar as evidências relacionadas com a formação de educadores ambientais, de acordo com as necessidades do conhecimento e as preocupações da comunidade que faz parte do seu campo de ação.

Palavras-chave: Formação; Educador ambiental; Conhecimento Didático do Conteúdo; Educação ambiental.

\section{Introdución}

La educación ambiental es un conocimiento que ha sido construido a partir de diferentes miradas epistemológicas, debido a que en sí misma interrelaciona diversas disciplinas y conocimientos, tales como: sociales, científicos, filosóficos, psicológicos, políticos y económicos; lo que hace que ésta tenga una identidad camuflada de muchos saberes. Lo real es que, frente a la crisis ambiental, este tema ha sido el eje de preocupación en distintos ámbitos profesionales y en diferentes reuniones mundiales, donde todos los gobiernos y entes políticos se preocupan por los aportes en el tema.

La educación ambiental como acción social influye, tanto en el educador ambiental como en su propio contexto de acción pedagógica. El papel de educador lo puede desempeñar cualquier persona que se encuentre en interacción con el otro, que permita la transmisión de un conocimiento, sin embargo, el actor ambiental se convierte en educador profesional cuando existe una reflexión de lo que enseña y su experiencia repercute en su propia historia y en la de aquel a quien enseña. Dicha reflexión, se identifica en su práctica diaria a través de planteamientos como: ¿Qué enseño?, ¿Para qué lo enseño?, ¿Qué características tiene eso que enseño?, ¿Qué me motiva para enseñar ese tema?, ¿Cómo el tema a enseñar está en mi vida?, estos aspectos y otros hacen la diferencia en la profesión docente.

Las diversas tendencias en educación ambiental dan paso a reformas y estructuras en la sociedad del conocimiento, es por eso que, que surge la necesidad de formar educadores ambientales. Inicialmente estaban muy involucrados con el tema los profesionales relacionados con los recursos naturales como los biólogos, ingenieros forestales, ingenieros ambientales, químicos, entre otras profesiones que estaban relacionadas con el manejo, el estudio y la conservación de los recursos naturales; estos actores, generaron gran cantidad de avances en investigación referentes a los tipos de ecologías y los impactos ambientales en diferentes campos de acción, con lo que el 
problema ambiental se fue identificando de carácter social, entonces se fue rescatando a la sociedad y quienes podían educar a ésta sociedad.

Los cuestionamientos entorno a la formación de educadores ambientales en diversos artículos y libros con enfoques epistemológicos y posturas teóricas diferentes, publican que la mayor producción de investigaciones está enfocada a la reflexión y divulgación de acciones de los mismos, en las cuales se describen pesquisas en un tema específico ambiental en su gran mayoría de problemas locales, proyectos de aula, reflexiones epistemológicas de las tendencias ambientales, historias ambientales conservacionistas, experiencias empresariales, interacción con la comunidad, entre otros temas.

Cabe resaltar que la educación ambiental tiene su origen en las políticas públicas ambientales, en la preocupación de alfabetizar, motivar y promover un conocimiento ambiental en la sociedad, que no sólo limitado a la comunidad de especialistas que están preocupados por las problemáticas ambientales, sino el involucrar a la sociedad en esa reconquista.

El Programa de las Naciones Unidas para el Medio Ambiente (PNUMA) generó un movimiento en relación a la formación y desarrollo de actividades, mencionando que: "En América Latina y el Caribe, La Red de Formación Ambiental para América Latina y el Caribe (RFA/ALC) nace en la década de los 80 a pedido de los gobiernos de la región al PNUMA, ante la necesidad de fortalecer las capacidades de los profesionales en la temática ambiental", dejando clara la intencionalidad de mejorar los procesos de formación y de capacitación en los educadores ambientales, para la divulgación y desarrollo de la educación ambiental en diferentes campos de acción.

El presente texto reconoce algunas investigaciones donde la educación ambiental es parte de esa formación, tanto en lo formal como en lo informal, desde diferentes enfoques y perspectivas, de acuerdo con el diálogo significativo de este conocimiento en el eje de acción de los educadores. Como lo afirma Loureiro $(2006$, p. 77 ) cuando interpreta la educación ambiental como un desafío entre lo particular y lo universal, donde el lenguaje entre lo diferente y lo similar constituyen el sentido del conocimiento que se está tejiendo hermenéuticamente en el diálogo de disciplinas y ciencias establecidas por comunidades históricamente reconocidas por la sociedad.

\section{Perspectivas en la Formación de Educadores Ambientales}

Para cumplir con las finalidades de la educación, es necesario compartir ciertos conocimientos, habilidades e ideales con el otro, el educar es poder mejorar unos a otros por medio del conocimiento, aprender continuamente y que hay cosas que pretenden ser sabidas (SAVATER, 1997).

En ese camino de la educación y el educar, de interacción entre educando y educador, es necesario partir del concepto de educador que en palabras de Savater (1997, p. 20) "es quien vivió antes el conocimiento y lo 
comparte con otros de una mejor manera, permitiendo que el otro reconozca elementos de su propia experiencia". Para Marques (2000), el educador tiene un papel social, movilizador de pensamientos, de acciones pedagógicas, de estructuras desafiantes en la sociedad, con formación específica en la acción educativa, construyendo un proceso permanente en el debate de cuestiones prácticas y teóricas en la educación.

El concepto de educador debe partir de su función social; los elementos de la enseñanza de la educación ambiental en la formación de estos profesionales que deciden ser constructores de un pensamiento ambiental en una sociedad dinámica y sujeta a cambios políticos, sociales, económicos, entre otros. La educación ambiental se encuentra en esa interacción con el otro, en la reflexión de las acciones, de pensamientos colectivos e individuales, el impacto de procesos políticos, económicos y personales. Por esto, todas las personas en algún momento de la vida somos educadores, aprendemos y enseñamos a otros a partir de la propia vida, experiencia y ejemplo.

De acuerdo con lo anterior surge la pregunta ¿En qué momento la educación ambiental dejara de ser responsabilidad de unos pocos y se convierte en responsabilidad de todos?, Se dará cuando el hombre entienda que él hace parte de los recursos de la tierra y que es el responsable de la interacción y movilización del pensamiento la lucha desde diferentes miradas ecológicas en contra de los desfases mundiales económicos, políticos, sociales que están atentando contra el equilibrio, ¿Qué pasaría si entre nosotros nos decapitamos y así eliminamos al otro de la esfera de la sociedad? sería una forma de destrucción de la tierra. Esto conlleva a pensar en la importancia de trabajar en conjunto, como afirma Barcelos (2012, p. 18, traducción nuestra) "[...] aprender a vivir juntos se constituye, hoy en una necesidad".

Por lo anterior, se hace necesario reflexionar y revisar que está aconteciendo con el enfoque conceptual de la educación ambiental desde sus contribuciones en la revisión de los elementos pedagógicos, didácticos, epistemológicos, históricos y del contexto de la educación ambiental en los programas de formación de educadores ambientales.

\section{Metodología}

Las definiciones anteriores, permiten la revisión de la direccionalidad de la "formación de educadores ambientales" desde las publicaciones en el tema. Se realizó un rastreo en revistas indexadas en bases de datos como: Dialnet, Scopus, Redalyc, Google académico, Scielo, BDTD, Elsevier, Latindex tanto en español como en portugués. Consultando documentos publicados entre los años 1998 y 2014 buscando representar y no generalizar el estado del conocimiento alrededor del concepto indagado en las publicaciones. Se orientó la búsqueda mediante las palabras clave y títulos de los artículos donde hicieran referencia a formación de educadores ambientales, se revisaron 45 artículos, de los cuales se profundizó en los que presentaban mayor 
descripción de las relaciones, acciones y perspectivas de acuerdo con la intencionalidad de formar en educación ambiental.

Al realizar el estudio de conocimiento alrededor de formación de educadores ambientales en revistas indexadas se pretendió responder al cuestionamiento en relación a los cuestionamientos como: ¿Sobre qué temas se está escribiendo e investigando en la formación de educadores y cómo estos están concibiendo la educación ambiental?, a partir del diálogo entre los artículos se interpreta esta intencionalidad para revisar el panorama de la formación de educadores ambientales y su relación con el conocimiento didáctico del contenido.

\section{Resultados y Análisis}

En la revisión y análisis de artículos se identificaron variedad de temas correspondientes a la formación de educadores ambientales, principalmente, basados en la relación de los profesores y la educación ambiental, representado desde el campo de acción en el ambiente escolar para promover e identificar perspectivas ambientales (saberes y acciones) en los jóvenes y niños, mediante proyectos de aula (ARAUJO, 2006; LIMA, 2012; GONZÁLEZ, 1998, entre otros).

Con relación a los aspectos tradicionales en la aplicación de la educación ambiental se rotula al profesor de ciencias naturales como el profesional más indicado para la generación de proyectos como lo evidenciaron Gil-Pérez et al. (2003). Al realizar un análisis de artículos publicados en revistas indexadas alrededor del tema educación ambiental se identificó que el tema está encasillado en los profesores de ciencias y en el argumento de ciencia, tecnología y sociedad.

Otras publicaciones comienzan a implementar el término de "educador ambiental" referenciando al profesor, y algunos autores solo enfocan al profesional de diferentes áreas de actuación como agente multiplicador del conocimiento en educación ambiental. En el cuadro 1, se resume el aporte de algunos artículos y temas que más se repiten:

En la búsqueda referente a la "formación de educadores ambientales" se observa en las publicaciones dos campos de acción, uno es la escuela y otro la universidad, ésta última como orientadora socio-histórica de la finalidad de educar a la sociedad ambientalmente.

Por lo tanto, la educación ambiental deja de estar sólo en las escuelas y pasa a la universidad, enmarcada en diferentes orientaciones curriculares, entonces la Universidad se considera como fuente principal de formación de los educadores ambientales. Consecuente con este interés, se observa en algunos artículos la relación hombre - naturaleza (TOZONI-REIS, 2002, p. 87) se mantiene en los currículos de formación inicial de profesores, , por otro lado, otros autores refuerzan la iniciativa de involucrar en dicho currículo otras propuestas como lo afirma Guerra \& Moser (2007), al asegurar la necesidad de 
los principios filosóficos y epistemológicos que fundamentan las propuestas de investigadores que actúan en educación ambiental, en publicaciones como la de Sauve (2004) deja clara la importancia de analizar la formación de educadores ambientales para verificar la necesidad de los educandos y relacionarla con su práctica para la restructuración curricular en la universidad.

Cuadro 1: Relación de aportes de los artículos y sus autores en formación de educadores ambientales.

\begin{tabular}{|c|c|}
\hline APORTE & AUTORES \\
\hline $\begin{array}{l}\text { Relación pensamiento del profesor y su práctica } \\
\text { como educador ambiental en el contexto escolar y la } \\
\text { concientización ambiental. }\end{array}$ & $\begin{array}{l}\text { Dias, 2012; Molon, 2008; } \\
\text { Queiroz, 2012; Figueiredo, } \\
\text { 2014, entre otros. }\end{array}$ \\
\hline $\begin{array}{l}\text { Estrategias de enseñanza en cursos de } \\
\text { formación de educadores. }\end{array}$ & $\begin{array}{l}\text { Freire, et al, 2013; Silva et } \\
\text { al, 2002; Sauve, } 2004 .\end{array}$ \\
\hline $\begin{array}{l}\text { El currículo en los Licenciados con la dimensión } \\
\text { socioambiental. }\end{array}$ & $\begin{array}{l}\text { Queiroz, 2012; Tozoni-Reis, } \\
2014 .\end{array}$ \\
\hline $\begin{array}{l}\text { Desarrollo de proyectos socioambiéntales } \\
\text { escolares y el conocimiento de lugar (escuela y la } \\
\text { comunidad). }\end{array}$ & Santos, 2013. \\
\hline $\begin{array}{l}\text { Comprender el sentido del significado de la } \\
\text { educación ambiental como forma de preservación, } \\
\text { conservación, concientización, y relaciones ecológicas y } \\
\text { biológicas en los educadores, mediante su lenguaje. }\end{array}$ & Pires y Francischett, 2014. \\
\hline $\begin{array}{l}\text { Materiales pedagógicos y las tecnologías en la } \\
\text { formación de profesores en educación ambiental e } \\
\text { instrumentos tecnológicos como medio de enseñanza. }\end{array}$ & $\begin{array}{c}\text { Guerra y Moser, 2008; } \\
\text { Moreira y Ramos, 2009; } \\
\text { Vigorito, 2011, Dias y Bonotto, } \\
2014 .\end{array}$ \\
\hline $\begin{array}{l}\text { El análisis del discurso en los profesores } \\
\text { alrededor del Licenciamiento Ambiental. }\end{array}$ & Santos et al, 2012. \\
\hline $\begin{array}{l}\text { La transdisciplinariedad en la construcción de } \\
\text { ambientes de práctica educativa, al igual que la } \\
\text { necesidad de ambientes de lectura y de escrita crítica. }\end{array}$ & $\begin{array}{l}\text { Farias, Galiazzi y Freitas } \\
\text { (2004). }\end{array}$ \\
\hline $\begin{array}{l}\text { En la formación de educadores ambientales } \\
\text { existe una dicotomía entre la formación en educación y la } \\
\text { formación ambiental, porque el último está lleno de } \\
\text { conceptos científicos y no pedagógicos, cuando los dos } \\
\text { buscan lo mismo, que es la formación de individuos. }\end{array}$ & Matos, 2009. \\
\hline
\end{tabular}

Fuente: Autores.

Dentro de la amplia temática de formación de educadores ambientales, entra un segundo subtema que es "ambientalización curricular" (MORA, 2012; RUSCHEINSKY et al., 2014), que involucra no sólo aspectos curriculares implícitos en los documentos educativos sino las mismas dinámicas universitarias, en el cuadro 2 se referencian algunas publicaciones que sustentan ese esbozo de información. 
Cuadro 2: Relación de artículos y aportes en ambientalización curricular como parte de la formación de educadores ambientales.

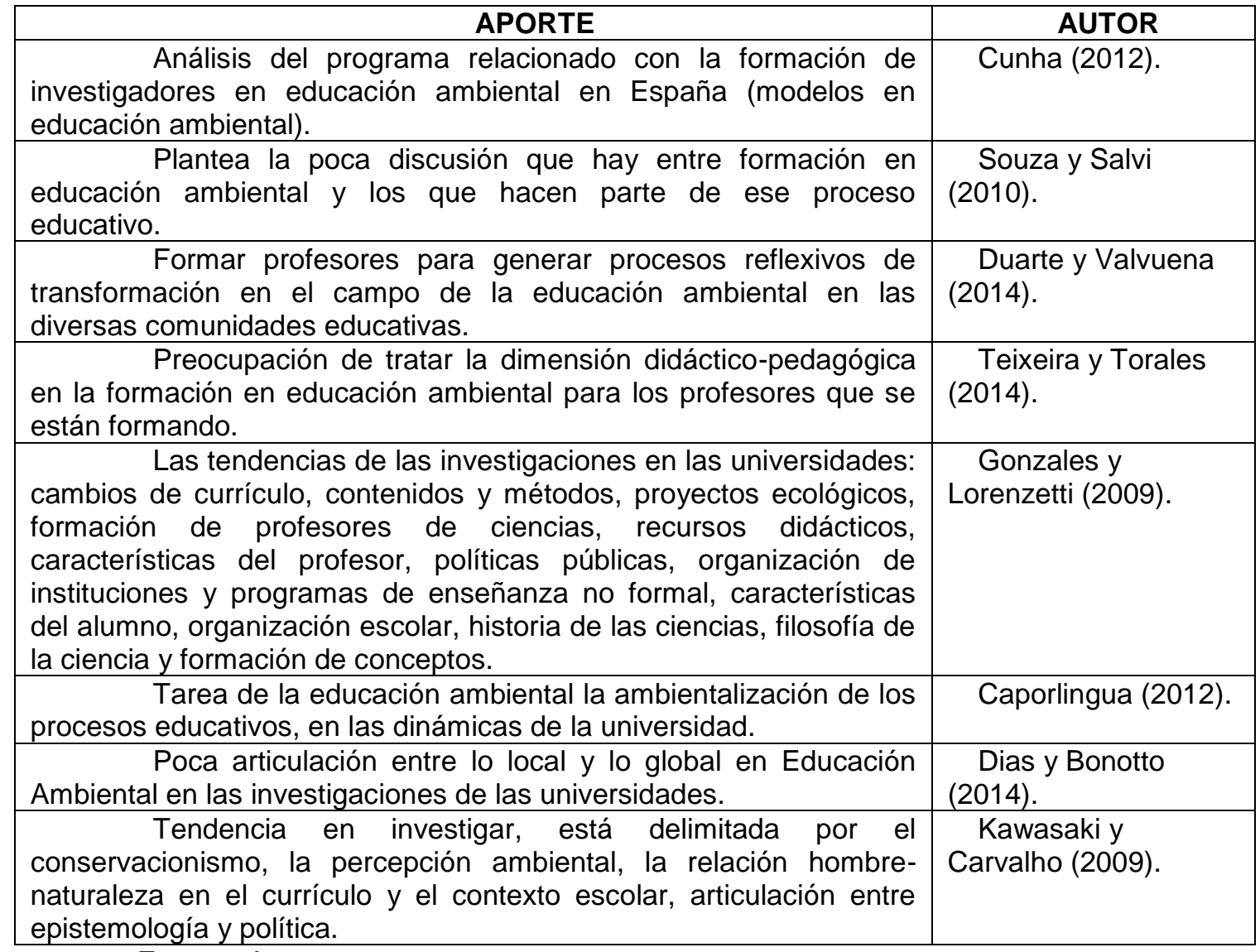

Fuente: Autores.

La formación en educación ambiental tiene como intencionalidad el educar, a su vez, la ciudadanía debe conocer las opciones para un mejor vivir, respetando los recursos naturales, a sí mismo y al otro, y al convivir entre una comunidad, con el fin de hacer que, este mundo sea mejor con una contemplación ambiental, donde no solo existe la relación hombre - naturaleza, sino hombre - sociedad, hombre y el otro. el ser humano en sus diferentes fases se involucra en muchos de los problemas actuales y que hacen parte de la destrucción de la tierra, debido al egoísmo del hombre y al buscar reconocimientos económicos, políticos, y sociales que pueden terminar afectando a unos y a otros.

\section{Un poco de discusión entre el Educador Ambiental y el Conocimiento Didáctico del Contenido}

Al reflexionar acerca del educador ambiental, Guimarães (2006), plantea la necesidad de repensar en los procesos que se llevan a cabo en las universidades para la formación continuada en educación ambiental, donde la investigación requiere un carácter más participativo en el espacio escolar y 
comunitario, generando investigación reflexiva en los procesos académicos en los cuales se encuentra el educador ambiental.

En la formación de educadores, existen elementos pedagógicos y didácticos que hacen que el currículo en esa formación no sea un esquema repetitivo, sino que cumpla una funcionalidad social. Según Marques (2000), esa formación requiere una reflexión de la educación: qué hacer y comprender qué se quiere de la educación, al igual que reconocer el diálogo entre las dimensiones de esa formación entre lo político, epistemológico, enseñanza, aprendizaje, la especificidad de los contenidos en los currículos, la identidad del educador en su desarrollo profesional y su historia de vida en el juego de relaciones pedagógicas y la responsabilidad social en la ciudadanía con emergentes acciones educativas.

Por lo tanto, esa formación desde la educación superior tiene estructuras que dependen de epistemologías individuales y colectivas en las cuales las finalidades del conocimiento pueden presentar desequilibrios en los campos de acción y en el desarrollo de cómo son visualizados los profesionales en educación ambiental, también desmotivaciones, inconformismos con su misma formación. Y en esa orientación de saberes, todos los agentes participantes en esa formación tanto profesores, estudiantes e instituciones, se convierten en objetivo de enseñabilidad, desde la interacción y el compartir intereses, experiencias, contextos teóricos y empíricos.

En consecuencia, el enseñar puede tener muchas preguntas y todas relacionadas con el conocimiento. Por ejemplo: ¿Qué quiero enseñar? ¿Qué quiero que aprendan? ¿Qué enseñar? ¿Cómo aprendí? ¿Cómo evaluar?, entre otras. De acuerdo con el conocimiento didáctico del Contenido (CDC), que es una de las líneas de investigación relacionadas con el pensamiento del profesor, inicialmente propuesto por Shulman Lee en 1982 como el conocimiento pedagógico del contenido "pedagogical contente knowledge", y ha sido analizado desde el mismo Shulman y traducido al español como "Conocimiento Didáctico del Contenido" por investigadores como Marcelo (1992), Bolívar, (2005), Parga, Mora y Martínez (2007), Acevedo (2009), entre otros. Generando variedad de interpretaciones y campos de análisis del pensamiento del profesional que enseña, a partir de la indagación en la enseñabilidad de disciplinas específicas, entre la crítica de la coherencia entre lo que se planea, se concibe y lo que se hace.

Se puede deducir que, la reunión de variables en esas posturas en relación del conocimiento pedagógico y el conocimiento que se enseña, se enmarcan en los que se denomina Conocimiento Didáctico del Contenido (CDC) como una interpretación y adaptación a las características del objetivo curricular desde lo que enseña, propuesta que puede ser definida de la siguiente forma, "se expresa como una emergencia de complexidad al integrar los cuatro grandes grupos de conocimiento (disciplinares, interdisciplinares, psicopedagógicos y contextualizados)" (PARGA; MORA, 2014, p. 333), donde se relacionan los elementos teóricos, procedimentales y actitudinales.

revista brasileira educação ambiental 
EI CDC entonces representa la correlación entre los conocimientos disciplinares pedagógicos y didácticos; al igual que las características del conocimiento de los estudiantes y del contexto educativo, de lo epistemológico e histórico del conocimiento a enseñar, con el propósito de ser más comprensibles y aplicables. Es un espacio de intersección interdisciplinar que contempla el currículo como un sistema dinámico. Conformado en su estructura por los componentes del conocimiento histórico-epistemológico (CHE), (comprender qué y cómo ha cambiado el conocimiento), del conocimiento disciplinar del contenido $(\mathrm{CdC})$, (comprender el área de conocimiento a enseñar) del conocimiento del contexto (Cc), (aprender a organizar el medio) y de los conocimientos psicopedagógico (CpP), (aprender a pensar en lo que se enseña desde la perspectiva del estudiante) (MORA; PARGA, 2008).

Con relación con la anterior definición, se puede pensar en una interpretación de acciones, ideales, saberes o conocimientos de diferente naturaleza en esa dinámica de comprensión de acciones y pensamiento de quien enseña y el lugar que puede este enseñar, bien sea en el ámbito formal o informal, en el proceso directo e indirecto de educar.

En el diálogo entre las teorías de aprendizaje y de enseñanza, son aspectos del currículo que en condiciones deseables el profesor o educador transita para cumplir con los objetivos de la educación ambiental, para contextualizar mediante la alfabetización a la sociedad en el cuidado, conservación de los recursos naturales y la aplicación de la misma, que en algunas circunstancias estas intencionalidades se ven afectadas por las condiciones sociopolíticas de los lugares donde se quiere realizar cambios y desarrollar propuestas socioambiéntales que permitan al educador acciones a favor de las intencionalidad de las políticas públicas ambientales. Desde la misma conservación de valores, respeto y tolerancia por el otro y ese reconocimiento de los saberes para desarrollar una sociedad sustentable desde los ejes ecológicos, sociales, axiológicos, humanísticos, políticos y científicos.

No solo es reflexionar en esa enseñanza, sino tener las capacidades para realizar asociaciones dialógicas entre la teoría y práctica, en la realidad y en las crisis sistémicas de la educación ambiental en diferentes campos de la sociedad. Entonces, el conocimiento ambiental puede ser reconocido como saber o puede ser parte de contenidos, de acuerdo con la postura teórica que se quiera analizar, describir o interpretar el pensamiento del profesor o del educador. Entre los aspectos curriculares del educador ambiental, pero no son fijos sino dinámicos, que caracterizan al profesional entre la coherencia de la educación ambiental, donde el hablar, escribir y las acciones que se desarrollan representan la simbología de interlocución entre su historia de vida, su pensar y su profesionalismo. 


\section{Conclusões}

En los ejes identificados en la revisión de publicaciones en formación de educadores ambientales, se presenta un panorama en relación a las acciones del profesor como educador ambiental en la escuela y en las comunidades, en relación a la ambientalización curricular, que refleja preocupación por estandarizar modelos, coherencia entre teoría y práctica del educador ambiental, procesos de transformación, dimensión didáctica pedagógica, articulación del currículo, incidencias en la comunidad, entre otros.

Se evidencia la necesidad de reconocer el lugar en la educación ambiental, es decir, resaltar los diálogos del conocimiento en favor de la constitución e identidad de la misma, para involucrar al contexto en los desarrollos de propuestas socioambiéntales, desde la iniciativa de los educadores para la alfabetización y concientización de la comunidad académica en el que están inmersos. Al igual que, la relación entre la educación ambiental y la formación de profesores es importante reconocer el lugar de ellos, así como todos los hombres no son iguales todos los lugares tampoco.

La interpretación del conocimiento del educador ambiental debe conocer su propio lugar de formación y de enseñanza, para así, conocer dónde se puede reestructurar o re-significar el currículo tanto explícito como implícito de la educación ambiental (transdisciplinar, multidisciplinar e interdisciplinar), e interpretar las razones de relación entre lo que se habla en educación ambiental, entre lo que se escribe y su acción en el aula y fuera de ella en relación con educación ambiental.

La relación de la educación ambiental en la formación de individuos, permite que el análisis desde el CDC sea especifico al contexto de la formación, teniendo en cuenta la orientación y la intencionalidad curricular, al igual que las institucionalidades que rigen la enseñanza de los programas que se ofrecen en la formación y las dinámicas de actuación y de generación de conocimiento.

\section{Referências}

ACEVEDO, J. Conocimiento didáctico del conocimiento para la enseñanza de la naturaleza de la ciencia II: una perspectiva. Revista Eureka, España, n. 6, v. 2., p. 164-189, 2009.

ARAUJO, D. Analise de um curso de formação docente utilizando as trilhas do Jardim Botânico de Porto Alegre /RS como espaço educador. 150 f. Dissertação (Mestrado em Ensino de Ciências e Matemática) - Universidade Luterana do Brasil, Canoas, 2006.

BARCELOS, V. Educação Ambiental: sobre princípios, metodologias e atitudes. Vozes: Petrópolis, 2012. 
BOLÍVAR, A. Conocimiento didáctico del contenido y didácticas específicas. En Currículum y formación del profesorado, v. 9, n. 2., p. 3-39, 2005.

BRASIL. Ministério do Meio Ambiente. Educação Ambiental Por um Brasil Sustentável: ProNEA. 4. ed. Brasília: MMA, 2014.112 p.

BRASIL. Ministério do Meio Ambiente. Programa Nacional de Educação Ambiental: ProNEA. 3. ed. Brasília: MMA, 2005. 102 p.

GIL-PEREZ, $D$ et al. A Educação cientifica e a situação do mundo: um programa de atividades dirigido a professores. Ciência \& Educação, Bauru, v. 9, n. 1, p.123-149, 2003.

GONGALEZ, E. Un enfoque antiesencialista centro y periférico de la educación ambiental. México: Mundi Prensa, 1998. 60 p.

GUERRA, A; MOSER, Salete. Materiais Pedagógicos e tecnologias: avanços e obstáculos na formação continuada em educação ambiental. Contrapontos, Itajaí, v. 8, n. 2, p. 253-269, oct. 2007.

GUIMARÃES, M. A Formação de educadores ambientais. 4. ed. Campinas: Papirus, 2006. $174 \mathrm{p}$.

LIMA, F.; MOTA, D. Educação ambiental e o educador ambiental: os desafios de elaborar e implantar projetos de educação ambiental nas escolas. Monografias Ambientais, Santa Maria, v. 7, n. 7, p. 1717-1722, mar. 2012. Disponible en: <cascavel.ufsm.br/revistas/ojs-2.2.2/index.../3338>. Recuperado: 10 mar. 2015.

LOUREIRO, C.F. Educar, participar e transformar em educação ambiental. Revista Brasileira de Educação Ambiental, São Paulo, v. 0, n. 0, p. 13-20, nov. 2004.

LOUREIRO, C.F. Trajetória e fundamentos da educação ambiental. 2. ed. São Paulo: Cortez, 2006.

MARCELO, C. Cómo conocen los profesores la materia que enseñan. Algunas contribuciones de la investigación sobre Conocimiento Didáctico del Contenido. In: VEZ, L.; MONTERO; J. M. (eds.). Las didácticas específicas en la formación del Profesorado (I). Santiago de Compostela: Tórculo, 1992. p 151-185.

MARQUES, M. Formação do Profissional da Educação. 3. ed. Ijuí: Unijui, 2000. $224 \mathrm{p}$.

MORA, W. Ambientalización curricular en la educación superior: un estudio cualitativo de las ideas del profesorado. Revista de Curriculum y Formación del Profesorado, España, v. 16, n. 2, p. 77-103, ago. 2012.

MORA, W; PARGA, D. De las tramas histórico/epistemológicas a las tramas de contexto/aprendizaje: un marco en el CDCC en química orgánica. Revista Tecné, Episteme y Didaxis. Bogotá. v.2 n. 24, p. 56 -81, 2008. 
MORA, W; PARGA, D. Aportes al CDC desde el pensamiento complejo. In: GARRITZ, A.; DAZA, S.; LORENZO, M. (Org.). Conocimiento Didáctico del Contenido: una perspectiva iberoamericana. [S.I.]: Editorial Académica Española, 2014. p 100- 143.

MORA, W; PARGA, D; MARTINEZ, L. El conocimiento didáctico del contenido como programa de investigación: un contexto para la enseñanza de la química. In: TED (Ed.), Tercer congreso internacional sobre formación de profesores de ciencias, número extra, comunicación oral ํㅡ. 97. Bogotá: TED, 2007.

PNUMA. Red de Formación Ambiental. [2013-2015?]. Disponible en: $<$ http://www.pnuma.org >. Recuperado: 04 abr. 2015.

QUINTAS, J. S. Considerações sobre a formação do educador para atuar no processo de gestão ambiental: Educação ambiental: desenvolvimento de cursos e projetos. São Paulo: Núcleo de Informações em Saúde Ambiental/USP: Signus, 2000.

RUSCHEINSKY, A. et al. Ambientalização nas instituições de educação superior no Brasil: caminhos trilhados, desafios e possibilidades. São Carlos: USP, 2014. $189 \mathrm{p}$.

SAUVE, L. Perspectivas curriculares para la formación de formadores en educación ambiental. In: Foro nacional sobre la incorporación de la perspectiva ambiental en la formación técnica y profesional, 1., 2004, México. Anais... San Luis de Potosí: Universidad Autónoma de San Luis de Potosí, 2004. p. 1 - 10.

SAUVE, L.; ORELLANA, I. La formación continuada de profesores en educación ambiental: la propuesta de Edamaz. Tópicos En Educación Ambiental, v. 4, n. 10, p. 50-62, 2002.

SAVATER, F. El valor de Educar. 2 edición. Ariel: Barcelona, 1997.

SHULMAN, L. Renewing the pedagogy of teacher education: the impact of subject-specific conceptions of teaching. In: Actas del Congreso "Las Didácticas específicas en la formación del profesorado". Santiago de Compostela: [s.n.], 1992.

TARDIF, M; LESSARD, C. O ofício de professor: história, perspectivas e desafios internacionais. Petrópolis: Vozes, 2008. 325 p.

TOZONI-REIS, M. Formação dos educadores ambientais e paradigmas em transição. Ciência \& Educação, Bauru, v. 8, n. 1, p. 83-96, nov. 2002. 